\title{
Impact of tricuspid valve insufficiency on the performance of left ventricular assist devices
}

\author{
Claudio J. R. Gomez Hamacher, ${ }^{a}$ Carolin Torregroza, MD, ${ }^{b}$ Najla Sadat, MD, ${ }^{a}$ Daniel Scheiber, MD, ${ }^{c}$ \\ Jil-Cathrin von der Beek, DVM, ${ }^{\mathrm{d}}$ Ralf Westenfeld, MD, PhD, ${ }^{\mathrm{c}}$ Ivonne Jeanette Knorr, DVM, ${ }^{\mathrm{d}}$ \\ Martin Sager, MD, PhD, ${ }^{\mathrm{d}}$ Artur Lichtenberg, $\mathrm{MD}, \mathrm{PhD},{ }^{\mathrm{a}}$ and Diyar Saeed, $\mathrm{MD}, \mathrm{PhD}^{\mathrm{a}, \mathrm{e}}$
}

\section{ABSTRACT}

Objective: To evaluate the impact of severe tricuspid valve insufficiency (TVI) at the time of left ventricular assist device (LVAD) implantation on the hemodynamic and LVAD parameters in an acute ovine model.

Methods: Stable heart failure (HF) was induced in 10 ovines through the application of $3 \pm 1$ coronary ligations. Once stable HF was obtained (after $15 \pm 5$ days), the animals were supported with an LVAD. Hemodynamic data and pump parameters were obtained and compared in 2 settings; first with LVAD in place after weaning from the cardiopulmonary bypass machine (no TVI condition) and second following the induction of severe TVI through resection of the tricuspid valve (TVI condition).

Results: There were no statistically significant differences in the hemodynamic and pump parameters between TVI condition and no TVI conditions except for lower cardiac output in the TVI condition (2 [1.38-2.8] L/min vs $3.2[1.55-3.7] \mathrm{L} / \mathrm{min}$, $P=.027)$ and the expected greater central venous pressure in the TVI condition (26 [24-31] $\mathrm{mm} \mathrm{Hg}$ vs 15 [13-25] mm Hg, $P=.020)$. A median pump flow of 2.8 (2.45-3.75) L/min versus $2.9(2.75-3.8) \mathrm{L} / \mathrm{min}$ in the TVI condition and no TVI condition was documented $(P=.160)$.

Conclusions: Results from this acute animal study suggest that severe TVI in HF with preserved right ventricular function does not have significant impact on the LVAD pump parameters. The observed reduction in cardiac output may warrant further investigations, especially under loading conditions. (JTCVS Open 2020;4:16-23)

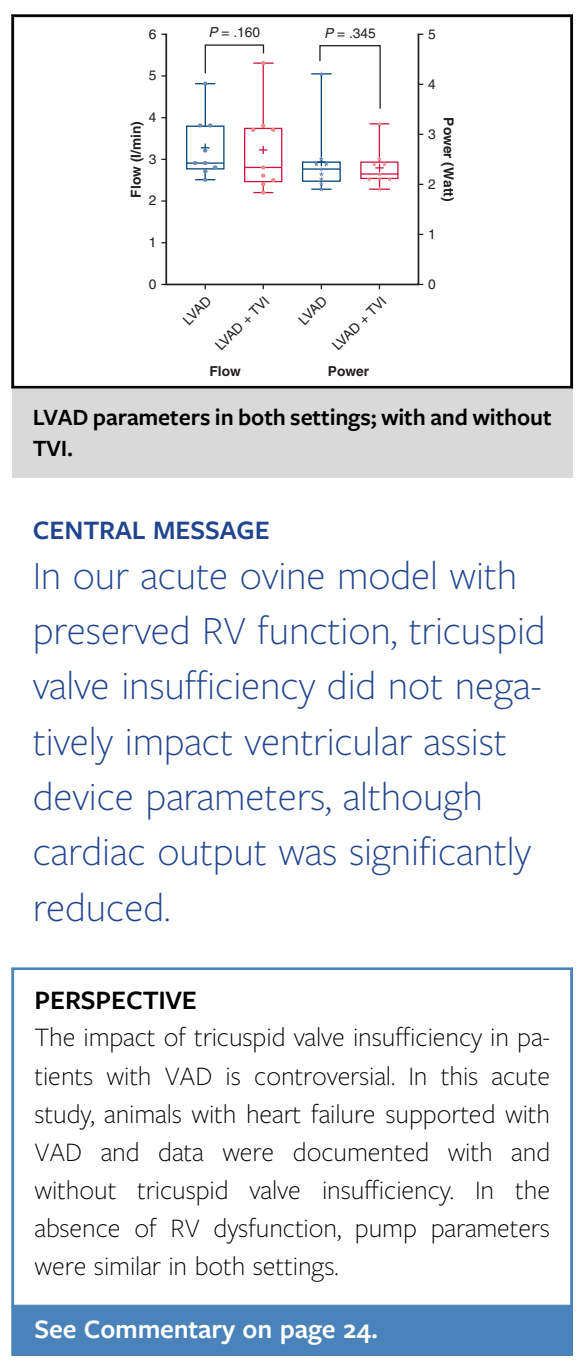

Heart failure (HF) is still one of the leading causes of death, affecting more than 26 million people worldwide. ${ }^{1}$ After the exhaustion of symptomatic therapies, heart transplantation and mechanical support systems are often ultimate resorts for the treatment of patients with end-stage HF. The increasing prevalence of patients with HF exceeds the number of organ donations, which has led to an increasing number of implantations of left ventricular assist devices

From the Departments of ${ }^{\mathrm{a}}$ Cardiovascular Surgery, and ${ }^{\mathrm{b}}$ Anesthesiology, ${ }^{\mathrm{c}}$ Division of Cardiology, Pneumology and Angiology, University Hospital, Heinrich-Heine University, Dusseldorf; ${ }^{\mathrm{d}}$ Central Unit for Animal Research and Animal Welfare Affairs, Heinrich-Heine University, Dusseldorf; and ${ }^{\mathrm{e}}$ University Department for Cardiac Surgery, Leipzig Heart Center, Leipzig, Germany.

This research was funded by "Forschungskommission KS 9772519" Heinrich Heine University Dusseldorf.

Received for publication Aug 28, 2020; accepted for publication Sept 18, 2020; available ahead of print Oct 23, 2020.

\footnotetext{
Address for reprints: Diyar Saeed, MD, PhD, University Department of Cardiac Surgery, Leipzig Heart Center, Strümpelstraße 39, 04289 Leipzig, Germany (E-mail: diyar.saeed@helios-gesundheit.de). 2666-2736

Copyright $(2020$ The Authors. Published by Elsevier Inc. on behalf of The American Association for Thoracic Surgery. This is an open access article under the CC BY-NCND license (http://creativecommons.org/licenses/by-nc-nd/4.0/). https://doi.org/10.1016/j.xjon.2020.09.001
} 


\author{
Abbreviations and Acronyms \\ $\mathrm{bpm}=$ beats per minute \\ $\mathrm{CO}=$ cardiac output \\ $\mathrm{CPB}=$ cardiopulmonary bypass \\ $\mathrm{CVP}=$ central venous pressure \\ $\mathrm{HF}=$ heart failure \\ $\mathrm{HR}=$ heart rate \\ LVAD $=$ left ventricular assist device \\ MAP $=$ mean arterial pressure \\ mPAP $=$ mean pulmonary artery pressure \\ PAPi $=$ pulmonary artery pulsatility index \\ $\mathrm{RV}=$ right ventricular \\ $\mathrm{RVF}=$ right ventricle failure \\ TVI = tricuspid valve insufficiency \\ TVR $=$ tricuspid valve reconstruction
}

Video clip is available online.

(LVADs) in recent years as either bridge to transplantation or destination therapy, improving patients' survival and quality of life..$^{2-5}$

Up to $50 \%$ of patients with end-stage HF may present with simultaneous tricuspid valve insufficiency (TVI). ${ }^{6}$ Due to a progressive failing of the left ventricle and the consequent congestion, the afterload of the right ventricle increases, resulting in TVI. Because TVI is associated with right $\mathrm{HF}$ and worse overall outcome, the presence of TVI raises the question whether tricuspid valve reconstruction (TVR) should be performed at the time of LVAD implantation. ${ }^{6,7}$ The current International Society of Heart and Lung Transplantation guidelines state that moderateto-greater TVI should prompt consideration of surgical repair at implantation. ${ }^{8}$ However, recent studies regarding treatment and outcome of TVI in patients with LVAD provide diverging results. ${ }^{9-16}$ Therefore, surgical approach varies among surgeons, and a consistent treatment strategy according to guidelines is missing. The aim of this study was to examine the direct impact of acute TVI at the time of LVAD implantation on the hemodynamic and LVAD parameters using an acute ovine model with stabile leftsided $\mathrm{HF}$ and preserved right ventricular (RV) function.

\section{METHODS}

All animals received humane care in compliance regarding "the EU Directive 2010/63," "the German Animal Welfare Act," and "the Animal Welfare Regulation Governing Experimental Animals," all of which are on par with "the Principles of Laboratory Animal Care" formulated by the National Society for Medical Research and the "Guide for the Care and Use of Laboratory Animals," prepared by the Institute of Laboratory Animal Resources and published by the National Institutes of Health (publication No. 86-23, revised 1996). The study protocol was approved by the corresponding regional authorities.

\section{Anesthesia and Surgical Procedures}

All animals received midazolam and atropine intramuscularly as premedication. After the induction of anesthesia using xylazine and ketamine (intramuscularly), endotracheal intubation was implemented, and animals were provided with a gastric tube. General anesthesia was continued using $1.3 \%$ isoflurane with oxygen air mixture, fentanyl, and pancuronium intravenously. Antiarrhythmic prophylaxis during induction of HF as well as LVAD implantation was administered intravenously before skin incision, before and after both coronary ligation, and LVAD insertion. The administered antiarrhythmic prophylaxis consisted of intravenous application of amiodarone, lidocaine, and magnesium. A continuous amount of glucose $5 \%$ or sodium chloride $0.9 \%$ solution at rates of 80 to $100 \mathrm{ml} / \mathrm{h}$ was given during the procedures. No blood products were given and no inotropic agents were administered to avoid arrhythmias. When necessary, intravenous norepinephrine was administered in moderate dose as an infusion to maintain a stable blood pressure. Notably, in all experiments, data were only obtained when stable conditions over several minutes were achieved to avoid variations due to fluid managements and/or vasoactive medications.

\section{Surgical Induction of Stable HF}

The study was carried out in 2 main stages. The first stage included induction of stable HF through coronary artery ligation. All animals underwent open-heart surgery using the left thoracotomy approach. Ovines were equipped with an arterial catheter in the left carotid artery, a central venous catheter in the left jugular vein, a pulmonary artery catheter, and an ultrasonic perivascular flow probe (AD Instruments, Dunedin, New Zealand) placed around the pulmonary artery for continuous cardiac output $(\mathrm{CO})$ measurement. Hemodynamic and echocardiographic data were obtained at baseline, after coronary ligation, and before LVAD implantation. Notably, stable HF was established through transmural ligation of diagonal and marginal branches only. The hemodynamic data consisted of mean arterial pressure (MAP), central venous pressure (CVP), mean pulmonary artery pressure (mPAP), heart rate (HR), and CO. Once animals showed echocardiographic, hemodynamic, and clinical signs of HF, the animals were brought back to the operating room and LVAD implantation surgery was conducted as the second-stage procedure. Notably, the actual LVAD flow was not measured with flow probes. The pump flow measurements were calculated flows (as it clinically used in patients with HeartWare HVAD [HeartWare Inc, Framingham, Mass]). However, the hematocrit values were adjusted every 10 minutes to achieve accurate calculated flows.

Similar to previous chronic animal studies of HF, HF in our studies was predefined as a $20 \%$ increase in pulmonary artery pressure and/or HR, and/ or $20 \%$ reduction in $\mathrm{CO}$ as well as echocardiographic findings of dilation of the left ventricle and/or $20 \%$ reduction of left ventricular ejection fraction.

\section{Surgical Procedure of LVAD Implantation and TVI Induction}

The second surgical procedure consisted of LVAD implantation and induction of TVI in animals with stable HF. All animals received heparin (300 IU/kg) for anticoagulation throughout the second surgery. Rightsided thoracotomy was performed in the fifth intercostal space and the pericardial sac was opened. The carotid artery and internal jugular vein were prepared for cannulation of cardiopulmonary bypass (CPB). A second venous cannula for $\mathrm{CPB}$ was inserted in the inferior vena cava. $\mathrm{CPB}$ was initiated and the LVAD was implanted. The correct placement of the LVAD was verified by epicardial echocardiography. All animals were supported with HeartWare LVAD (HeartWare Inc). Figure 1 shows the LVAD 


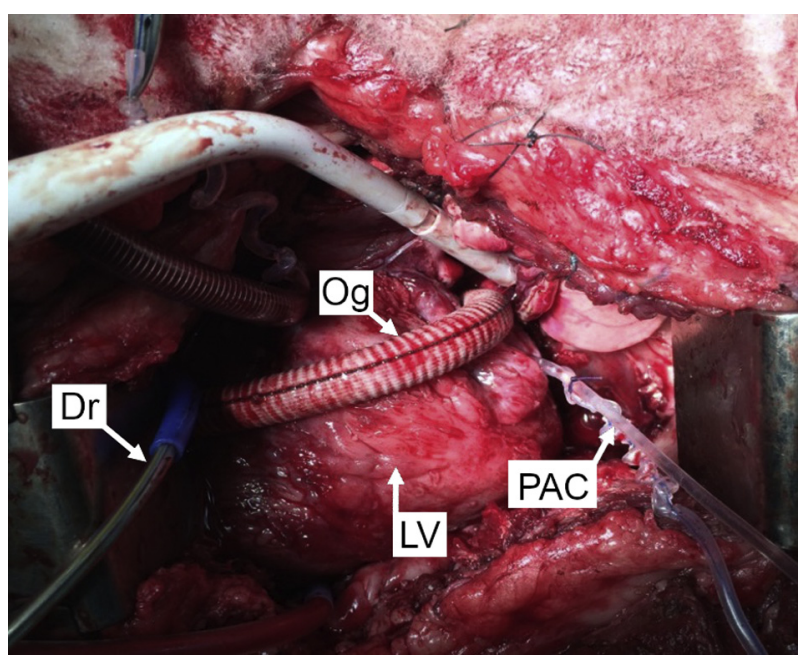

FIGURE 1. Intraoperative setting, left ventricular assist device in place. $D r$, Driveline; $O g$, outflow graft; $L V$, left ventricle; $P A C$, pulmonary artery catheter.

in place. The aforementioned hemodynamic data and LVAD parameters (pump flow, speed, and power consumption) were obtained in 2 conditions, first, after weaning of CPB (no TVI condition) with LVAD in place and second, after reinitiating $\mathrm{CPB}$, induction of TVI through surgical resection of the tricuspid valve (TV), and weaning off CPB (TVI condition). The pump speed was kept unchanged to allow reasonable comparison between both conditions. Figure 2 shows TV resection procedure. Once data were obtained, the animals were killed humanely in the operating room after completion of surgical procedure using pentobarbital-sodium. For the first stage of the study, hemodynamic and echocardiographic data were compared between all animals at baseline, post-ligation, and before LVAD implantation. In the second stage of the study, the hemodynamic and LVAD parameters at TVI and no TVI condition were compared within the same animal.

\section{Statistical Analysis}

Statistical analysis was performed using SPSS for Mac (Version 25; IBM Corp, Armonk, NY). As not all variables were distributed normally

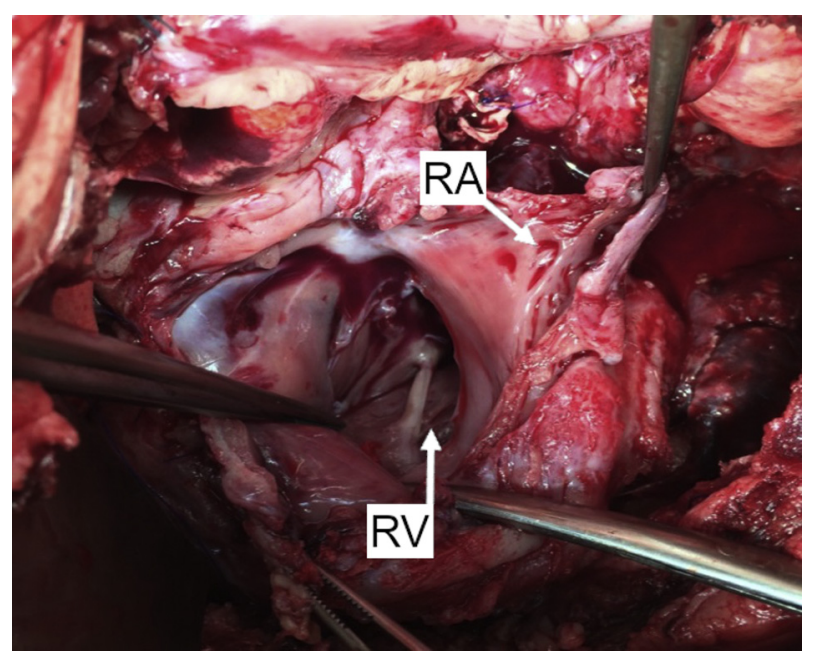

FIGURE 2. Resection of the tricuspid valve. $R A$, Right atrium; $R V$, right ventricle. (tested by Shapiro-Wilk test), data evaluation was achieved through a Wilcoxon signed-rank test (nonparametric paired $t$ test). Data are reported as median value (interquartile range).

\section{Primary Outcomes}

Primary outcomes are hemodynamic changes between LVAD no TVI and LVAD with TVI setting, specifically CVP, MAP, CO, and mPAP, as well as LVAD parameters, including flow, pump speed, and power consumption.

\section{RESULTS}

Ten female ovines (mean weight $61 \pm 7 \mathrm{~kg}$ ) underwent surgical induction of HF through ligation of diagonal and marginal branches. A total of $3 \pm 1$ transmural ligations were used. Stable HF was established after $15 \pm 5$ days. Animals showed clinical signs of HF, including reduced general conditions, wheezing, tachypnea, and dyspnea, on day $8 \pm 3$ post-ligation. Main findings in hemodynamic data were a significant increase in HR $(72 \pm 9$ beats per minute $[\mathrm{bpm}]$ and $100 \pm 28 \mathrm{bpm}, P=.01)$ and mPAP $(15 \pm 4 \mathrm{~mm} \mathrm{Hg}$ and $18 \pm 5 \mathrm{~mm} \mathrm{Hg}, P=.034)$ at baseline and before LVAD implantation. Further, diastolic pulmonary pressure increased from $9 \pm 4 \mathrm{~mm} \mathrm{Hg}$ to $15 \pm 3 \mathrm{~mm}$ $\mathrm{Hg}(P=.018)$. Repeated echocardiography showed a drastic decrease in the left ventricular ejection fraction from baseline to latest measurements as well as a significant dilation in the left ventricle shown through an increase in left ventricular end-systolic and left ventricular enddiastolic volume. The left ventricular ejection fraction decreased significantly from $63 \pm 13 \%$ (baseline) to $43 \pm 6 \%$ (latest) $(P=.012)$, whereas left ventricular end-diastolic volume and left ventricular end-systolic volume both increased from $55 \pm 12 \mathrm{~mL}$ (baseline) to $70 \pm 22 \mathrm{~mL}$ (latest) $(P=.03)$ and $22 \pm 5 \mathrm{~mL}$ (baseline) to $42 \pm 16 \mathrm{~mL}$ (latest) $(P=.022)$, respectively.

Meanwhile, right ventricle function was assessed using echocardiography and through calculating the pulmonary artery pulsatility index (PAPi), a marker for RV dysfunction, at baseline and previous LVAD implantation. The right ventricle function was described in echocardiography as normal in all animals, and PAPi data revealed no significant change $(0.91 \pm 0.63$ at baseline vs $1.15 \pm 0.56$ in stable HF; $P=.39$ ) indicating that right ventricle function was not impaired at the time of LVAD implantation.

All 10 animals underwent second surgery for LVAD implantation and TVI induction. The animals were connected to CPB for a total of $101 \pm 22$ minutes and the total LVAD support time was $41 \pm 20$ minutes. Results comparing the hemodynamic parameters in no TVI condition to the parameters in TVI condition are shown in Figures 3 and 4. Changes in HR, MAP, and mPAP were not significant when comparing the 2 groups. HR showed no changes with 95 (82-127) bpm in no TVI and 98 (87-122) bpm in TVI condition $(P=.953)$. MAP also remained stable at 64 (60-74) $\mathrm{mm} \mathrm{Hg}$ (no TVI) and 68 (63-71) $\mathrm{mm} \mathrm{Hg}$ 


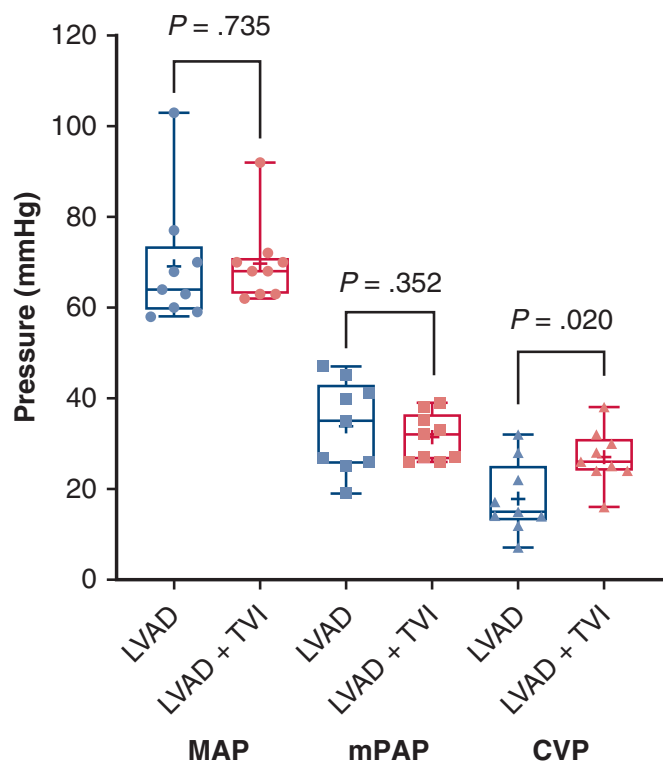

FIGURE 3. Changes in hemodynamic data in both conditions (no TVI versus TVI). $L V A D$, Left ventricular assist device; TVI, tricuspid valve insufficiency; $M A P$, mean arterial pressure; $M P A P$, mean pulmonary artery pressure; $C V P$, central venous pressure.

(TVI) $(P=.735)$. Similarly, no major changes in the mPAP was observed (35 [26-43] $\mathrm{mm} \mathrm{Hg}$ in no TVI and 32 [27-37] $\mathrm{mm} \mathrm{Hg}$ in TVI condition, $P=.352$ ). As expected, a statistically significant increase of the CVP was observed from 15 (13-25) $\mathrm{mm} \mathrm{Hg}$ in no TVI to 26 (24-31) $\mathrm{mm} \mathrm{Hg}$ in the TVI condition $(P=.020)$. Furthermore, the $\mathrm{CO}$ decreased from $3.2(1.55-3.7) \mathrm{L} / \mathrm{min}$ in no TVI to $2 \mathrm{~L} / \mathrm{min}(1.38-2.8)$ in the TVI condition $(P=.027)$.

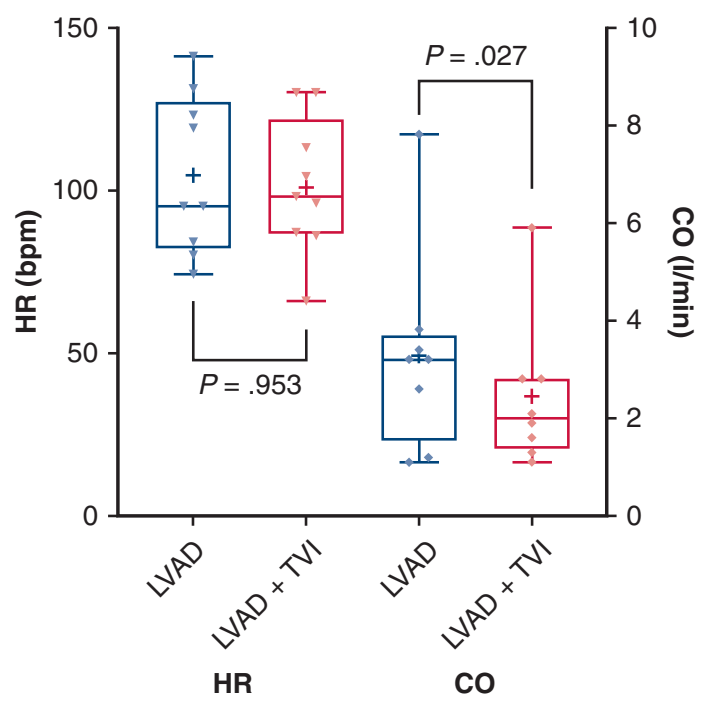

FIGURE 4. Changes in hemodynamic data. $\mathrm{CO}$ and HR in both conditions. $H R$, Heart rate; $C O$, cardiac output; $L V A D$, left ventricular assist device; $T V I$, tricuspid valve insufficiency.

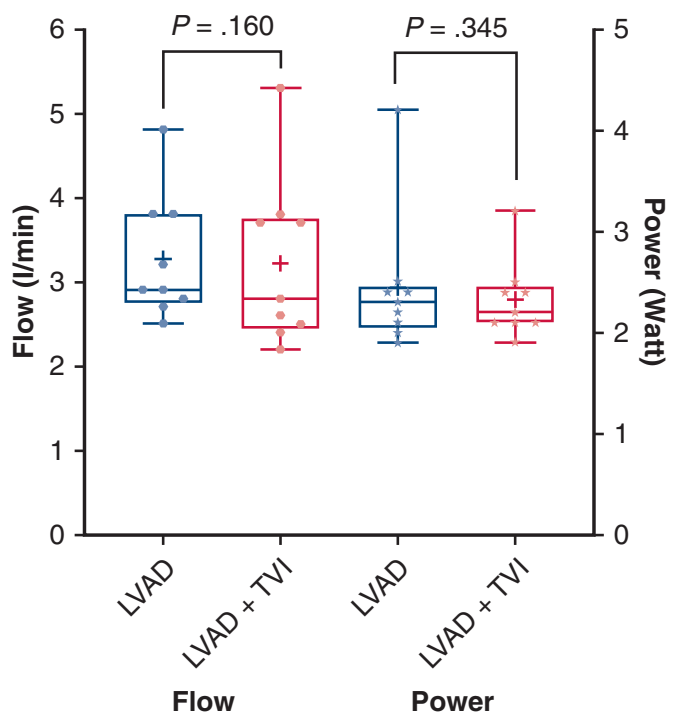

FIGURE 5. Changes in LVAD parameters in both settings; TVI versus no TVI. LVAD, Left ventricular assist device; TVI, tricuspid valve insufficiency.

There were no significant changes displayed in the LVAD parameters (Figure 5). A median LVAD pump speed of 2300 (2200-2400) revolutions per minute (no TVI condition) and 2300 (2200-2400) revolutions per minute (TVI condition) was documented $(P=.317)$. Meanwhile, a median LVAD flow of $2.9(2.75-3.8) \mathrm{L} / \mathrm{min}$ (no TVI condition) and $2.8(2.45-3.75) \mathrm{L} / \mathrm{min}$ (TVI condition) was obtained $(P=.160)$. The median LVAD power consumption was 2.3 (2.05-2.45) Watt (W) (no TVI condition) and 2.2 (2.12.45) W (TVI condition) $(P=.345)$ (Figure 6, $A-D)$. A summary of the hemodynamic and LVAD parameters is portrayed in Table 1.

\section{Comment}

In this acute animal setting of stable HF and severe iatrogenic TVI, no significant differences in the pump parameters were observed between TVI condition and no TVI condition. Further, the hemodynamic parameters stayed stable in both conditions except for a decrease in $\mathrm{CO}$ without negative impact on the pump parameters.

Functional or secondary TVI is the most frequent form of TVI. ${ }^{9,17}$ It can develop as a consequence of either single or biventricular failure or, although less frequently, of high pulmonary vascular pressure, all resulting in dilation of the annular ring. Further, implantation of an implantable cardioverter/defibrillator or pacemaker can also lead to a hindrance of valve leaflet coaptation. ${ }^{17,18}$ All of the aforementioned factors can be observed in patients with endstage HF requiring LVAD support. It has been described that LVAD implantation alone improves RV function, thereby leading to reduced TVI severity, which would render TVR obsolete. ${ }^{19-21}$ The improvement of the RV 

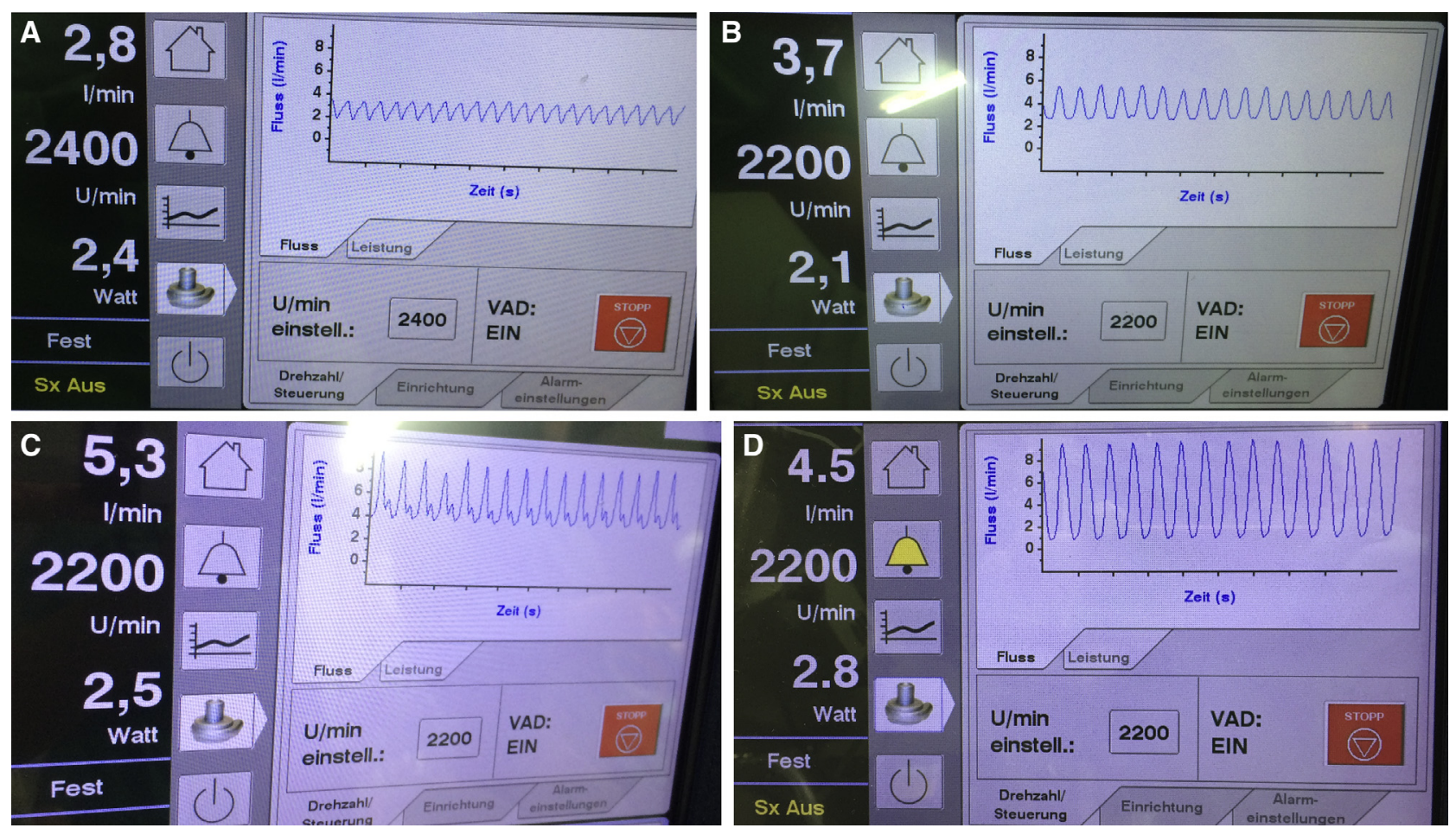

FIGURE 6. A-D, Intraoperative photographs of left ventricular assist device (LVAD) monitors depicting LVAD parameters and pulsatility in tricuspid valve insufficiency condition. Pulsatility was not analyzed qualitatively or included in the results. L/min, Liters per minute; U/min, revolutions per minute; Fluss, flow; Zeit, time; s, seconds; VAD, ventricular assist device.

function is mostly due to better unloading of the left ventricle after LVAD implantation, resulting in a decrease of pulmonary vascular pressure and therefore of the RV afterload. $^{22} \mathrm{RV}$ dysfunction may improve over time after LVAD implantation and better tricuspid valve competence may be achieved. ${ }^{21,23}$ Meanwhile, it has also been reported that LVAD implantation may also lead to a leftward deviation of the interventricular septum, particularly if run at

TABLE 1. Comparison of hemodynamic and LVAD parameters in both settings (no TVI vs TVI condition)

\begin{tabular}{lccc}
\hline \multicolumn{1}{c}{ Variable } & LVAD $^{*}$ & LVAD + TVI* & $\boldsymbol{P}$ value $\dagger$ \\
\hline HR, bpm & $95(82-127)$ & $98(87-122)$ & .953 \\
CO, L/min & $3.2(1.55-3.7)$ & $2(1.38-2.8)$ & $.027 \ddagger$ \\
MAP, mm Hg & $64(60-74)$ & $68(63-71)$ & .735 \\
mPAP, mm Hg & $35(26-43)$ & $32(27-37)$ & .352 \\
CVP, mm Hg & $15(13-25)$ & $26(24-31)$ & $.020 \ddagger$ \\
Flow, L/min & $2.9(2.75-3.8)$ & $2.8(2.45-3.75)$ & .160 \\
Power, W & $2.3(2.05-2.45)$ & $2.2(2.1-2.45)$ & .345 \\
Speed, rpm & $2300(2200-2400)$ & $2300(2200-2400)$ & .317 \\
\hline
\end{tabular}

$L V A D$, Left ventricular assist device; TVI, tricuspid valve insufficiency; $H R$, heart rate; $C O$, cardiac output; $M A P$, mean arterial pressure; $M P A P$, mean pulmonary artery pressure; $C V P$, central venous pressure; rpm, revolutions per minute. *Data are expressed as median (interquartile range). $\dagger$ Wilcoxon signed-rank test. $\ddagger P<.05$ statistically significant. greater speed mode, which might possibly cause an exacerbation of the TVI. ${ }^{24}$

Considering the association between TVI and right ventricle failure (RVF), the presumption that concomitant tricuspid valve procedures along with LVAD implantation could possibly contribute to minimizing the chance of RVF is postulated. ${ }^{6}$ While it has been demonstrated that concomitant tricuspid valve procedure does not increase the risk of operative mortality and early postoperative morbidity, performing either TVR or replacement does not affect the clinical outcome in patients with LVAD. ${ }^{25,26}$ Piacentino and colleagues showed that TVR reduces right $\mathrm{HF}^{27-29}$ Furthermore, they describe a prolonged CPB time in the TVR cohort although shorter hospitalization. However, a difference in mortality between the 2 groups was not detected.

The question whether to perform TVR is heavily debated and controversial. Recently, mostly single-center studies have come to disparate conclusions. ${ }^{9-16}$ Saeed and colleagues ${ }^{9}$ conducted a retrospective analysis of patients undergoing LVAD implantation with or without TVR. The main finding of this study was that TVR led to prolonged CPB time and greater requirements of blood products, thus concluding that omitting TVR is reasonable in VAD candidates. Veen and colleagues ${ }^{30}$ have recently carried out a meta-analysis of 8 publications. They showed that 


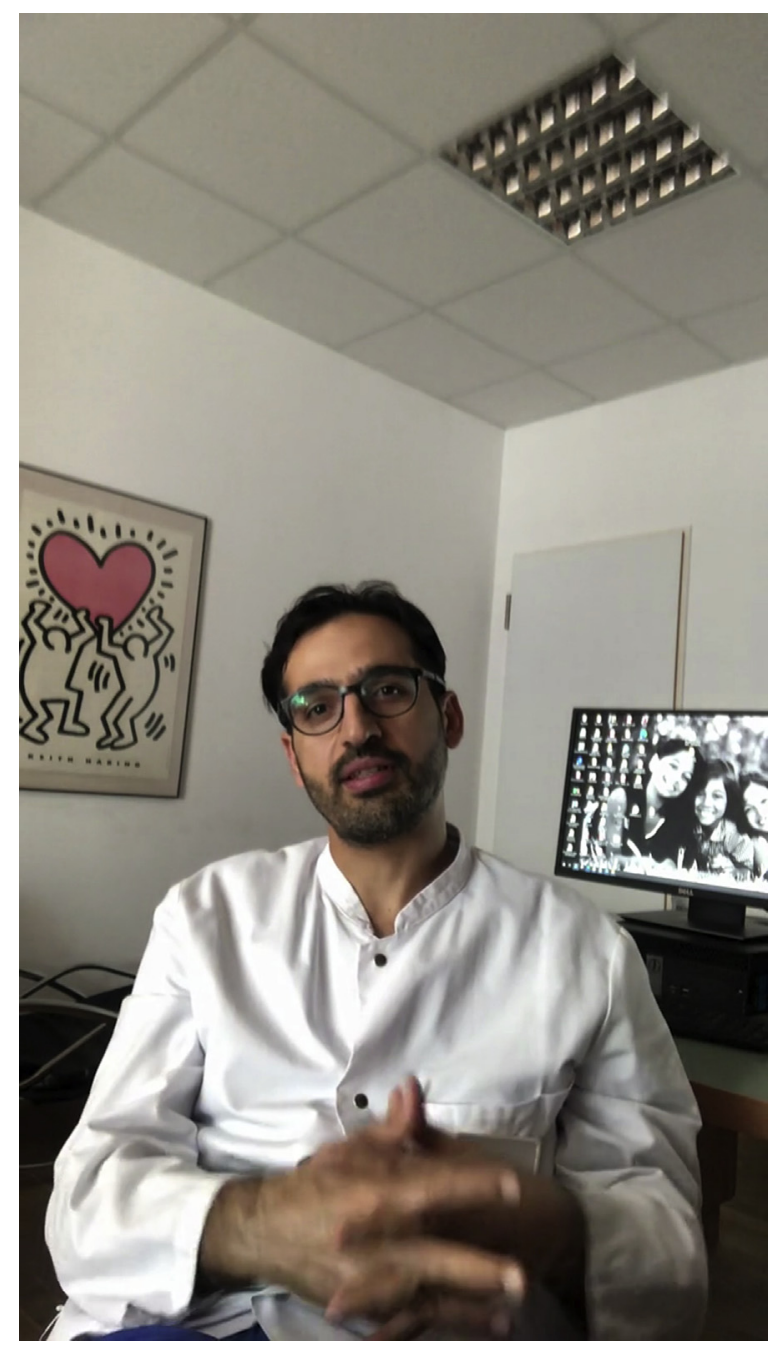

VIDEO 1. The senior author explains the concept, importance, and relevance of this study. Video available at: https://www.jtcvs.org/article/ S2666-2736(20)30107-8/fulltext.

concomitant TVR during LVAD implantation provides similar results in late mortality and RVF when compared with LVAD implantation alone. Furthermore, they failed to show any significant differences in early mortality, RVF, acute kidney failure, hospital stay, and need for RV assist device. The systematic review by Dunlay and colleagues ${ }^{31}$ focused on early outcomes supports the findings of Veen and colleagues. The database analyses of INTERMACS (Interagency Registry for Mechanically Assisted Circulatory Support), EUROMACS (European Registry for Patients with Mechanical Circulatory Support), and the Society of Thoracic Surgeons have led to comparable results. ${ }^{7,32,33}$ These database analyses show that TVR is associated with worse early postoperative outcomes but do not affect surgical mortality. ${ }^{33}$ Furthermore, TVR led to significantly improved TVI shortly after TVR, but differences in probability of TVI disappeared between the TVR and the non-TVR group over a period of time. ${ }^{7,32}$ In addition, TVR does not show a survival benefit; thus, TVR may not be beneficial. ${ }^{32}$ Meanwhile, Robertson and colleagues $^{33}$ found longer hospital stays and a significantly greater risk of renal failure in the TVR group and raised the question that the decision for TVR should not solely be based on echocardiographic findings. In summary, the majority of currently available literature show that TVR at the time of LVAD implantation may not be necessary.

Our study is the first animal and translational research study dealing with this controversial topic (Video 1). We have shown that the induction of severe iatrogenic TVI in stable HF ovines with preserved right ventricle function is feasible in acute settings. We appreciate that establishing an animal model of $\mathrm{HF}$ that exactly simulates patients with end-stage $\mathrm{HF}$ is challenging. ${ }^{34}$ We intentionally chose a model that preliminarily causes left ventricular failure without major impact on the RV function. We believe that severely reduced RV function and functional TVI may rather benefit from better preconditioning before the pump implantation procedure (aggressive diuresis, etc.) and/or biventricular support strategy in emergent cases than TVR. That being said, we hypothesize that moderate TVI without severe RV dysfunction, such as those caused by defibrillator leads, may eliminate the need for TVR. We postulate that adding TVR procedure may not provide any advantage. ${ }^{9}$

In this study, we were able to establish stable HF with hemodynamic and echocardiographic parameters mimicking HF. In the second-stage procedure, severe acute TVI was induced through surgical resection of the TV. All hemodynamic parameters remain stable in the group with LVAD alone or LVAD with TVI except for expected greater CVP value in the TVI group (15 [13-25] $\mathrm{mm} \mathrm{Hg}$ vs 26 [24-31] $\mathrm{mm} \mathrm{Hg}, P=.020)$ and lower $\mathrm{CO}$ in the TVI group (3.2 [1.55-3.7] $\mathrm{L} / \mathrm{min}$ vs 2 [1.38-2.8] $\mathrm{L} / \mathrm{min}, P=.027)$. The increase of CVP was expected as a result from the TV resection. The decrease of the CO could possibly be due to the second initiation of the $\mathrm{CPB}$, which was conducted to perform the resection of the valve resulting in prolonged $\mathrm{CPB}$ time. The duration and re-initiation of the CPB cause additional immunologic and operative trauma, which may explain this finding. Another explanation of the reduction in CO may be the fact that left ventricular filling may be better in the absence of TVI leading to more CO. Notably, the $\mathrm{RV}$ output, which was measured using ultrasonic flow probes, represents total $\mathrm{CO}$ whereas pump flow only reflects the flow through the pump. That being said, we assume that in the TVI condition, the majority of the $\mathrm{CO}$ went through the pump. Meanwhile, in the no TVI condition the remaining $\mathrm{CO}$ went through the native aortic valve. Interestingly, the performance of the LVAD remains similar in both conditions. An average pump flow of $2.9(2.75-3.8) \mathrm{L} / \mathrm{min}$ in the no TVI group versus $2.8(2.45-3.75) \mathrm{L} / \mathrm{min}$ in the TVI 
group was documented $(P=.160)$. This finding may indicate that having TVI per se without major RV dysfunction may not negatively impact the pump performance. Needless to say, the duration of the experiments was not long enough to allow investigating the impact of chronic TVI on the outcome.

Limitations of this study are similar to any other animal study of HF, as a one-to-one model simulating patients with end-stage HF is challenging. However, the changes in the diastolic pulmonary pressures and echocardiography findings indicated severe HF in our study. Further, we believe that our model was representative enough to show the effect of severe TVI in the setting of continuous flow pumps. Notably, all of these animals had preserved RV function. Therefore, our results may be limited to those VAD candidates with TVI and preserved RV function such as those caused by pacing leads. Furthermore, the validity of this acute model is limited to the short period of observation time as well as the rather short follow-up time after induction of HF. In addition, the setting of an acute iatrogenic TVI might not reflect the pathophysiological changes occurring in patients with chronic TVI over time, which makes drawing a conclusion about clinical practice difficult. Therefore, we plan to repeat this study in a chronic model with an increased number of animals and randomized in comparable TVI and no TVI condition cohorts in the near future. In fact, we already performed 2 successful chronic experiments. One ovine survived LVAD implantation. However, the study was terminated after 5 days due to LVAD pump thrombosis. Another ovine survived LVAD implantation with TV resection but died afterwards due to respiratory insufficiency. Additional limitation includes the fact that no detailed echocardiographic measurement of the right ventricle was performed before LVAD implantation. Nevertheless, all animals had a good right ventricular function based on PAPi data and echocardiographic observation at the time of LVAD procedure. The induced HF was limited to the left ventricle in these animals. We hypothesize that presence of TVI with preserved RV function would not impact the VAD parameters. Another limitation is the fact that we were comparing data of the same animals in 2 different conditions. The presumably negative impact of second round of $\mathrm{CPB}$ on $\mathrm{CO}$ might have been eliminated if we would have had performed either LVAD or LVAD with TVI. A true matched control would have had a re-initiation of CPB in a second group of animals and sham right atriotomy in the no TVI condition. However, this would have necessitated more animals and more resources. Needless to say, another limitation is the fact that the pump flows were calculated flows and even updated hematocrit values may not guarantee real-time flow measurements.

In conclusion, our data suggest that TVI in subjects with HF with preserved RV function did not negatively impact
LVAD parameters. The observed reduction in CO may warrant further investigation, especially considering loading conditions. Further chronic experiments as well as clinical trials are needed to conclusively clarify the necessity of TVR in patients with LVAD. However, results from this study could possibly encourage omitting the TVR procedure, particularly in LVAD candidates with preserved RV function and moderate TVI and/or TVI due to pacing leads.

\section{Conflict of Interest Statement}

The authors reported no conflicts of interest.

The Journal policy requires editors and reviewers to disclose conflicts of interest and to decline handling or reviewing manuscripts for which they may have a conflict of interest. The editors and reviewers of this article have no conflicts of interest.

\section{References}

1. Ambrosy AP, Fonarow GC, Butler J, Chioncel O, Greene SJ, Vaduganathan M, et al. The global health and economic burden of hospitalizations for heart failure lessons learned from hospitalized heart failure registries. J Am Coll Cardiol. 2014;63:1123-33

2. Benjamin EJ, Virani SS, Callaway CW, Chamberlain AM, Alexander MR, Susan S, et al. Heart disease and stroke statistics - 2018 update. A report from the American Heart Association. Circulation. 2018;137:67-492.

3. Pozzi M, Giraud R, Tozzi P, Bendjelid K, Robin J, Meyer P, et al. Long-term continuous-flow left ventricular assist devices (LVAD) as bridge to heart transplantation. J Thorac Dis. 2015;7:532-42.

4. Slaughter MS. Long-term continuous flow left ventricular assist device support and end-organ function: prospects for destination therapy. J Card Surg. 2010; 25:490-4.

5. Slaughter MS, Rogers JG, Milano CA, Russell SD, Conte JV, Feldman D, et al. Advanced heart failure treated with continuous-flow left ventricular assist device. N Engl J Med. 2009:361:2241-51.

6. Piacentino V III, Williams ML, Depp T, Garcia-Huerta K, Blue L, Lodge AJ, et al. Impact of tricuspid valve regurgitation in patients treated with implantable left ventricular assist devices. Ann Thorac Surg. 2011;91:1342-7.

7. Song HK, Gelow JM, Mudd J, Chien C, Tibayan FA, Hollifield K, et al. Limited utility of tricuspid valve repair at the time of left ventricular assist device implantation. Ann Thorac Surg. 2016;101:2168-74.

8. Feldman D, Pamboukian SV, Teuteberg JJ, Birks E, Lietz K, Moore SA, et al. The 2013 International Society for Heart and Lung Transplantation guidelines for mechanical circulatory support: executive summary. J Hear Lung Transplant. 2013; 32:157-87.

9. Saeed D, Kidambi T, Shalli S, Lapin B, Malaisrie SC, Lee R, et al. Tricuspid valve repair with left ventricular assist device implantation: is it warranted? J Hear Lung Transplant. 2011;30:530-5.

10. Brewer RJ, Cabrera R, El-Atrache M, Zafar A, Hrobowski TN, Nemeh HM, et al. Relationship of tricuspid repair at the time of left ventricular assist device implantation and survival. Int J Artif Organs. 2014;37:834-8.

11. Fujita T, Kobayashi J, Hata H, Seguchi O, Murata Y, Yanase M, et al. Right heart failure and benefits of adjuvant tricuspid valve repair in patients undergoing left ventricular assist device implantation. Eur J Cardiothorac Surg. 2014; 46:802-7.

12. Han J, Takeda K, Takayama H, Kurlansky PA, Mauro CM, Colombo PC, et al. Durability and clinical impact of tricuspid valve procedures in patients receiving a continuous-flow left ventricular assist device. J Thorac Cardiovasc Surg. 2016; 151:520-7.e1.

13. Krishan K, Nair A, Pinney S, Adams DH, Anyanwu AC. Liberal use of tricuspidvalve annuloplasty during left-ventricular assist device implantation. Eur J Cardiothorac Surg. 2012;41:213-7.

14. Maltais S, Topilsky Y, Tchantchaleishvili V, Mckellar SH, Durham LA, Joyce LD, et al. Surgical treatment of tricuspid valve insufficiency promotes early reverse remodeling in patients with axial-flow left ventricular assist devices. J Thorac Cardiovasc Surg. 2012;143:1370-6. 
15. Oezpeker C, Zittermann A, Paluszkiewicz L, Piran M, Puehler T, Sayin AO, et al. Tricuspid valve repair in patients with left-ventricular assist device implants and tricuspid valve regurgitation: propensity score-adjusted analysis of clinical outcome. Interact Cardiovasc Thorac Surg. 2015;21:741-7.

16. Zhigalov K, Szczechowicz M, Mashhour A, Kadyraliev BK, Mkalaluh S, Easo J, et al. Left ventricular assist device implantation with concomitant tricuspid valve repair: is there really a benefit? J Thorac Dis. 2019;11(suppl 6): $902-12$.

17. Taramasso M, Vanermen H, Maisano F, Guidotti A, La Canna G, Alfieri O. The growing clinical importance of secondary tricuspid regurgitation. J Am Coll Cardiol. 2012;59:703-10.

18. Chang JD, Manning WJ, Ebrille E, Zimetbaum PJ. Tricuspid valve dysfunction following pacemaker or cardioverter-defibrillator implantation. J Am Coll Cardiol. 2017;69:2331-41.

19. Lee S, Kamdar F, Madlon-Kay R, Boyle A, Colvin-Adams M, Pritzker M, et al. Effects of the HeartMate II continuous-flow left ventricular assist device on right ventricular function. J Hear Lung Transplant. 2010;29:209-15.

20. Morgan JA, Paone G, Nemeh HW, Murthy R, Williams CT, Lanfear DE, et al. Impact of continuous-flow left ventricular assist device support on right ventricular function. J Hear Lung Transplant. 2013;32:398-403.

21. Kukucka M, Potapov E, Stepanenko A, Weller K. Acute impact of left ventricular unloading by left ventricular assist device on the right ventricle geometry and function: effect of nitric oxide inhalation. J Thorac Cardiovasc Surg. 2011; 141:1009-14.

22. Klotz S, Deng MC, Stypmann J, Roetker J, Wilhelm MJ, Hammel D, et al. Left ventricular pressure and volume unloading during pulsatile versus nonpulsatile left ventricular assist device support. Ann Thorac Surg. 2004;77:143-9.

23. Atluri P, Fairman AS, MacArthur JW, Goldstone AB, Cohen JE, Howard JL, et al. Continuous flow left ventricular assist device implant significantly improves pulmonary hypertension, right ventricular contractility, and tricuspid valve competence. J Card Surg. 2013;28:770-5.

24. Holman WL, Bourge RC, Fan P, Kirklin JK, Pacifico AD, Nanda NC. Influence of left ventricular assist on valvular regurgitation. Circulation. 1993;88:II309-18.

25. Pal JD, Klodell CT, John R, Pagani FD, Rogers JG, Farrar DJ, et al. Low operative mortality with implantation of a continuous-flow left ventricular assist device and impact of concurrent cardiac procedures. Circulation. 2009;120(suppl 1):215-9.

26. Deo SV, Hasin T, Altarabsheh SE, McKellar SH, Shah IK, Durham L, et al. Concomitant tricuspid valve repair or replacement during left ventricular assist device implant demonstrates comparable outcomes in the long term. J Card Surg. 2012;27:760-6.

27. Milano C, Pagani FD, Slaughter MS, Pham DT, Hathaway DR, Jacoski MV, et al. Clinical outcomes after implantation of a centrifugal flow left ventricular assis device and concurrent cardiac valve procedures. Circulation. 2014;130(suppl 1):3-11.

28. John R, Naka Y, Park SJ, Sai-Sudhakar C, Salerno C, Sundareswaran KS, et al. Impact of concurrent surgical valve procedures in patients receiving continuous-flow devices. J Thorac Cardiovasc Surg. 2014;147:581-9.

29. Piacentino V III, Ganapathi AM, Stafford-Smith M, Hsieh MK, Patel CB Simeone AA, et al. Utility of concomitant tricuspid valve procedures for patients undergoing implantation of a continuous-flow left ventricular device. J Thorac Cardiovasc Surg. 2012;144:1217-21.

30. Veen KM, Muslem R, Soliman OI, Caliskan K, Kolff EA, Dousma D, et al. Left ventricular assist device implantation with and without concomitant tricuspid valve surgery: a systematic review and meta-analysis. Eur J Cardiothorac Surg. 2018;54:644-51.

31. Dunlay SM, Deo SV, Park SJ. Impact of tricuspid valve surgery at the time of left ventricular assist device insertion on postoperative outcomes. ASAIO J. 2015;61: $15-20$.

32. Veen KM, Caliskan K, de By TMMH, Mokhles MM, Soliman OI, Mohacsi P, et al. Outcomes after tricuspid valve surgery concomitant with left ventricular assist device implantation in the EUROMACS registry: a propensity score matched analysis. Eur J Cardiothorac Surg. 2019;56: 1081-9.

33. Robertson JO, Grau-Sepulveda MV, Okada S, O’Brien SM, Brennan JM, Shah AS, et al. Concomitant tricuspid valve surgery during implantation of continuous-flow left ventricular assist devices: a Society of Thoracic Surgeons database analysis. J Hear Lung Transplant. 2014;33:609-17.

34. Zahr R, Saeed D, Fumoto H, Horai T, Shalli S, Anzai T, et al. A pilot study for inducing chronic heart failure in calves by means of oral monensin. Int J Biomed Sci. 2010;6:1-7.

Key Words: heart failure, ventricular assist device, tricuspid valve insufficiency, tricuspid regurgitation, animal model, ovine model, coronary ligation 